\section{Olanzapine-induced neutropenia}

\author{
Kirti Malhotra, ${ }^{1}$ Priscilla Vu, ${ }^{1}$ \\ Danielle H. Wang, ${ }^{1}$ Hank Lai, ${ }^{2}$ \\ Lawrence R. Faziola2 \\ 1School of Medicine, 2Department \\ of Psychiatry, University of California \\ Irvine, Orange, CA, USA
}

\section{Abstract}

Olanzapine-induced neutropenia is a rare adverse effect that is currently poorly described in literature. Although neutropenia is a known adverse effect of clozapine, it has been associated with the use of other antipsychotic medications like olanzapine. This case report describes and reviews a case of olanzapine-induced neutropenia in a schizophrenic patient. Although the mechanism of antipsychotic-induced neutropenia is still debated, this report attempts to discuss current theories as well as supply evidence in literature of this rare but potentially dangerous adverse effect.

\section{Introduction}

Olanzapine is a second-generation antipsychotic for treatment of schizophrenia and bipolar disorder. Second generation antipsychotics like olanzapine first came into use in the 1990s and have widespread use due to presumed higher efficacy, less extrapyramidal side effects and fewer adverse drug interactions.1,2 Although known to produce side effects such as weight gain and metabolic syndrome, a lesser studied phenomenon associated with Olanzapine therapy is granulocytopenia. ${ }^{3-5}$ This phenomenon is usually associated with Clozapine, a second-generation antipsychotic. 6 Several mechanisms have been proposed as being responsible for the neutropenia generated by these drugs. One mechanism suggests that these drugs may modulate levels of granulocyte colony stimulating factor (GCSF). G-CSF is noted to be involved in granulocytopenias. It is expressed at enhanced levels during increased granulocyte counts. ${ }^{6,7}$ In clozapine and olanzapine-induced granulocytopenia, G-CSF was not detectable in plasma. ${ }^{6}$ Although the exact mechanism of interaction between these typical antipsychotics and GCSF is not clear, the relationship warrants further study. A second proposed mechanism for clozapine-induced neutropenia is the formation of nitrenium cations catalyzed by Flavincontaining monoxenage-3 (FM03) system of leukocytes. ${ }^{8,9}$ Although classified as a thienobenzodiazepine, olanzapine has a simi- lar chemical structure and pharmacological profile to clozapine, thus, it is likely that its mechanism of action in inducing neutropenia is similar to that of clozapine. 10

This case study provides an example and discussion of a patient with schizophrenia experiencing neutropenia suspected to be caused by olanzapine. The purpose of this report is to evaluate the relationship between olanzapine, drug-induced neutropenia, and other contributing factors and to inform clinicians of such possible side effects when considering use of olanzapine.

\section{Case Report}

A 27-year-old healthy Caucasian male with a history of schizophrenia was hospitalized for severe exacerbation of psychosis. He was hospitalized for 87 days and during this time he was treated with olanzapine, fluoxetine, ziprasidone, valproate, lurasidone, and fluphenazine. Thirty-two weeks prior to admission, the patient had 2 ED visits over the course of two weeks while on olanzapine 15-20 mg daily with white blood cell (WBC) $5.9 \times 109 / \mathrm{L}$ downtrend to $5.0 \times 10^{9} / \mathrm{L}$ and absolute neutrophil counts (ANC) $4.9 \times 109 / \mathrm{L}$ to $2.3 \times 109 / \mathrm{L}$ (WBC reference range $4.0-10.5 \times 109 / \mathrm{L}$; ANC reference range $2.0-8.1 \times 109 / \mathrm{L})$. On admission, he was started on olanzapine $15 \mathrm{mg}$ with a WBC of $3.5 \times 10^{9} / \mathrm{L}$ and his ANC was $1.4 \times 10^{9} / \mathrm{L}$, within the category of mild neutropenia (ANC $>1000$ and $<1500$ cells $/ \mu \mathrm{L}$ ). The patient did not report adverse events on this medication. He noted stabilization of mood and improved psychotic symptoms. Olanzapine was titrated up to $40 \mathrm{mg}$ at day 20 . The patient did well for the following weeks while hospitalized, with an addition of fluoxetine $10 \mathrm{mg}$ daily as an adjunct for treatment of depression on hospital day 28 and increased to $20 \mathrm{mg}$ on hospital day 37. Forty-one days into his hospitalization, the patient's WBC count remained low.

Two months into his hospitalization, patient began responding to internal stimulus, endorsed auditory hallucinations, and became hyper-religious in thought process. At hospital day 60 , the patient's WBC and ANC remained low (Figure 1). Clozapine was initially discussed as a potential treatment option but as the patient's ANC was below initiating parameters $\left(\mathrm{WBC} \geq 3.5 \times 10^{9} / \mathrm{L}\right.$ and $\mathrm{ANC} \geq 2.0 \times 10^{9} / \mathrm{L}$ are acceptable for initiating clozapine), a trial of clozapine was excluded. Ziprasidone, was noted to have been effective for treatment of psychosis in the patient's mother who also has schizophrenia and thus he was started on ziprasidone $40 \mathrm{mg}$ (bis in die) BID on hospital day 60 with the intent to cross-taper olanzapine. However, patient reported feeling restless on ziprasidone and no longer wished to contin-
Correspondence: Lawrence Richard Faziola, Department of Psychiatry and Human Behavior, University of California Irvine, Building 3, Route 88, 101 The City Drive Orange, CA 92868, USA. Tel.: +1.714.456.7304 - Fax: + 1.714.456.5112 E-mail: lfaziola@uci.edu

Key words: Olanzapine; antipsychotic; neutropenia; granulocytopenia; adverse effects.

Contributions: the authors contributed equally.

Conflict of interest: the authors declare no potential conflict of interest.

Received for publication: 13 February 2015 Accepted for publication: 13 February 2015.

This work is licensed under a Creative Commons Attribution NonCommercial 3.0 License (CC BYNC 3.0).

(C) Copyright K. Malhotra et al., 2015

Licensee PAGEPress, Italy

Mental Illness 2015; 7:5871

doi:10.4081/mi.2015.5871

ue the cross-taper. Olanzapine was thus returned to $40 \mathrm{mg}$. Sixty-two days into hospitalization, the patient's WBC and ANC declined (Figure 1). On hospital day 64, the patient was also started on valproate ER $1500 \mathrm{mg}$ nightly for mood stabilization. His valproic acid serum level was measured to be at a therapeutic level of $81 \mathrm{mg} / \mathrm{L}$ on day 67 (therapeutic range from 50 to $100 \mathrm{mg} / \mathrm{L}$ ). At hospital day 73, follow-up WBC remained low and his ANC trended down (Figure 1). Patient was also noted to have decreasing sleep and appeared euphoric, hyper-religious and grandiose. These symptoms were thought to be fluoxetine-induced and it was titrated off on hospital day 75 . At this time, he was also started on lurasidone 40 $\mathrm{mg}$ with the intent to titrate off olanzapine due to $15 \mathrm{~kg}$ weight gain since admission. Lurasidone was titrated to $80 \mathrm{mg}$ and olanzapine was decreased from $40 \mathrm{mg}$ to $30 \mathrm{mg}$. However, patient's psychotic symptoms worsened, therefore lurasidone was discontinued and the patient was started on fluphenazine 5 mg BID on hospital day 82. Four days later fluphenazine was increased to $10 \mathrm{mg}$ BID for persistent psychotic symptoms. At discharge, patient was psychiatrically stable and discharged to a residential treatment center with olanzapine $30 \mathrm{mg}$ nightly, valproate ER 1500 $\mathrm{mg}$ nightly, and fluphenazine $10 \mathrm{mg}$ BID.

\section{Discussion}

The patient is a 27 year old Caucasian male 
with no known history of hematologic disease or previous episodes of antipsychotic-induced granulocytopenia or neutropenia. Additionally, he did not exhibit symptoms of infection and did not require any special medical treatment or work-up.

There are many causes that may induce neutropenia including psychotropic medications and illicit substances. Although there has not been a clear link between Fluoxetine and neutropenia, there have been case reports of fluoxetine-induced neutropenia.11,12 However, this patient was neutropenic prior to starting fluoxetine and his WBC and ANC levels did not appear to change with higher doses of fluoxetine. Therefore, fluoxetine was an unlikely source of this patient's neutropenia.

Valproate does have a known risk to hematologic dysfunction including neutropenia. However, the addition of valproate to the patient's regimen did not reveal a further decrease in WBC and only showed a slight decrease in ANC from $1.7 \times 109 / \mathrm{L}$ to $1.6 \times 109 / \mathrm{L}$. Thus, valproate is less likely to be the predominant contributor to the patient's hematologic depression. Another potential cause of the patient's neutropenia may have been related to his history of substance use. Upon admission, the patient admitted to using methamphetamine five days prior to admission, marijuana twice in the previous month, and injecting heroin three times in the previous month. His drug screen panel on admission was positive for amphetamines. And while there is no clear causal link between illicit substances and neutropenia, there are several case reports as well as a surveillance study reporting neutropenia due to use of cocaine or heroin contaminated with levamisole (ergamisole), ${ }^{13}$ a hydrochloride salt with antihelmithic and immunomodulatory property. The patient was not evaluated for Levamisole in his urine upon admission, was only mildly neutropenic during his hospitalization and otherwise asymptomatic. As a result, it is unclear whether contamination of illicit substances may have played a role in this patient's neutropenia or the concurrent use of heroin or amphetamine with olanzapine may have potentiated neutropenia.

Prior to admission and throughout his hospitalization, the patient was being maintained on olanzapine. As the patient was neutropenic early in his admission, it is possible his home medication of olanzapine may have caused hematologic depression prior to admission. It is convincing that while on olanzapine in the hospital, the patient remained slightly hematologically depressed with WBC and ANC levels below reference range. Therefore, it is likely that the patient's hematologic depression was due to his Olanzapine use. Although multiple attempts had been tried to titrate off of Olanzapine to observe any normalization of his WBC and ANC, his WBC and ANC levels increased only slightly during his hospitalization but remained below reference range. Should his WBC and ANC have continued to rise while on Olanzapine, this finding would have been consistent with case reports of transient granulocytopenia with spontaneous resolution despite continued olanzapine treatment. 13

There are several case reports of olanzapine-induced neutropenia.14,15 Two hypothesized risk factors are a previous history of antipsychotic-induced neutropenia/ agranulocytosis (including clozapine-induced neutropenia/agranulocytosis), and a dose-dependent effect of antipsychotic-induced hemato- logic depression. 14 The mechanism for olanzapine's effect on hematologic function is currently unknown, however due to chemical structural similarities to clozapine, the mechanisms are thought to be similar. ${ }^{16}$

\section{Conclusions}

This case report represents evidence of neutropenia induced by olanzapine use. Although poorly described in literature, olanzapineinduced neutropenia is a potentially serious side effect that should be considered with the

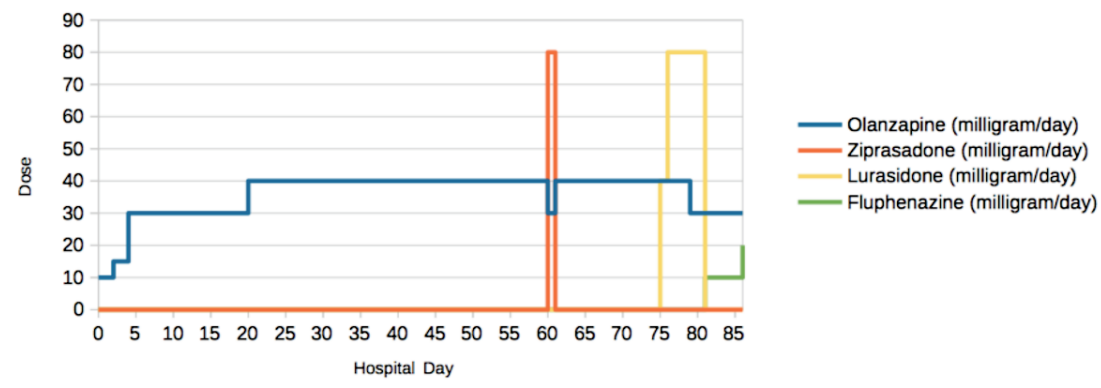

Non-antipsychotic Medication Changes during Hospitalization

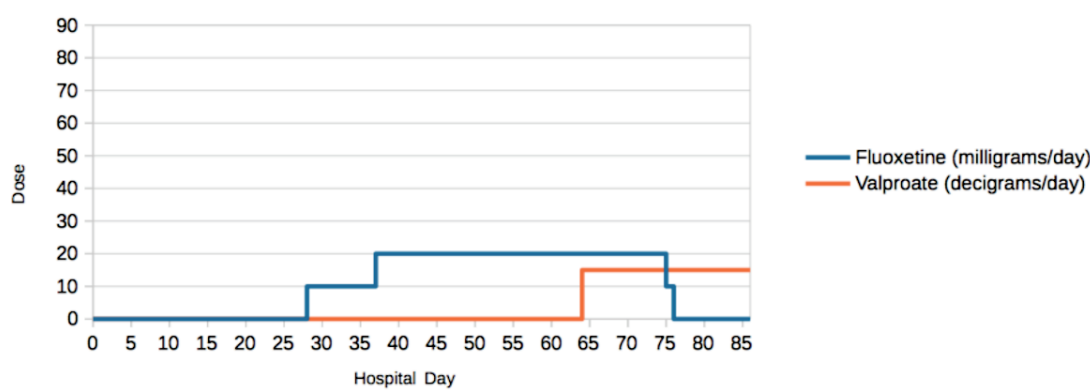

Absolute Neutrophil Count and WBC Changes

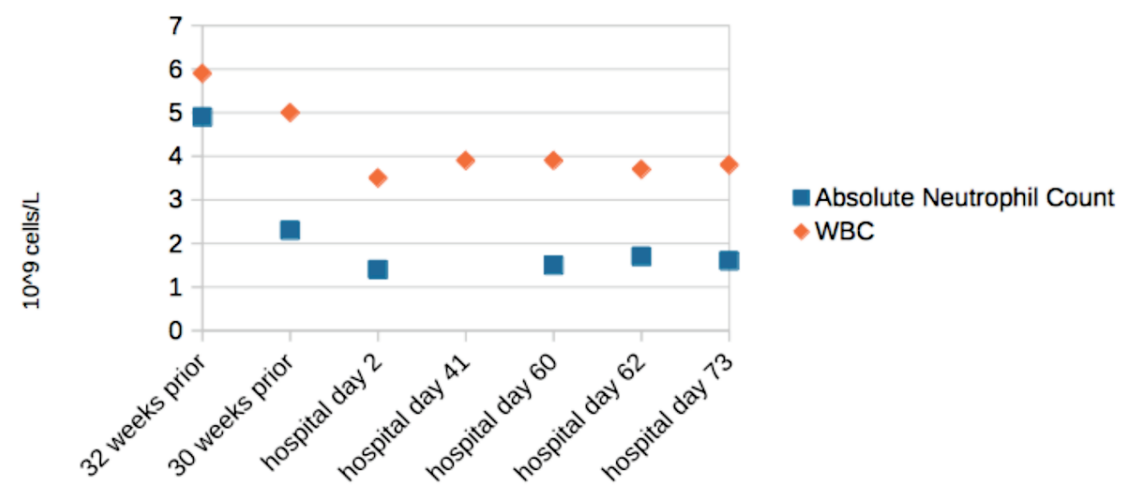

Figure 1. Treatment course of the medications and dosing associate with white blood cell and absolute neutrophil counts in the patient admitted for psychosis with home medication of olanzapine. 
use of olanzapine. Further studies are needed to investigate the mechanism and risk factors for olanzapine-induced neutropenia. One risk factor to be considered may be concurrent use of heroin or amphetamine contaminated with Levamisole with olanzapine. ${ }^{13}$ Additionally, there are currently no guidelines for examining WBC and ANC levels with olanzapine dosing, but this case emphasizes the importance of monitoring $\mathrm{CBC}$ at higher dosages of olanzapine.

\section{References}

1. Lieberman JA. Comparative effectiveness of antipsychotic drugs. A commentary on: Cost Utility Of The Latest Antipsychotic Drugs In Schizophrenia Study (CUtLASS 1) and Clinical Antipsychotic Trials of Intervention Effectiveness (CATIE). Arch Gen Psychiatry 2006;63:1069-72.

2. Lieberman JA, Stroup TS, McEvoy JP, et al. Effectiveness of antipsychotic drugs in patients with chronic schizophrenia. N Engl J Med 2005;353:1209-23.
3. Benedetti F, Cavallaro R, Smeraldi E. Olanzapine induced neutropenia after clozapine-induced neutropenia. Lancet 1999;354:567.

4. Meissner W, Schmidt T, Kupsch A, et al. Reversible leukopenia related to olanzapine. Mov Disord 1999;5:872-3.

5. Steinwachs A, Grohmann R, Pedrosa F, et al. Two cases of olanzapine-induced neutropenia. Pharmacopsychiatry 1999;32: 154-6.

6. Schuld A, Kraus T, Hinze-Selch D, et al. Granulocyte colony-stimulating factor plasma levels during clozapine- and olanzapine-induced granulocytopenia. Acta Psychiatr Scand 2000;102:153-5.

7. Pollmacher T, Fenzel T, Mullington J, Hinze-Selch D. The influence of clozapine treatment on plasma granulocyte colonystimulating (G-CSF) levels. Pharmacopsychiatry 1997;30:118-21.

8. Flanagan RJ, Dunk L. Haematological toxicity of drugs used in psychiatry. Hum Psychopharmacol 2008;23:27-41.

9. Sikora A, Adamus J, Marcinek A. Disproportionation of clozapine radical: a link between one-electron oxidation of clozapine and formation of its nitrenium cation. Chem Res Toxicol 2007;20:1093-8.

10. Nooijen PM, Carvalho F, Flanagan RJ. Haematological toxicity of clozapine and some other drugs used in psychiatry. Hum Psychopharmacol 2011;26:112-9.

11. Vilinsky FD, Lubin A. Severe neutropenia associated with fluoxetine hydrochloride. Ann Intern Med 1997;127:573-4.

12. Bavle A. Fluoxetine-induced neutropenia. Indian J Psychiatry 2012;54:388-9

13. Vagi SJ, Sheikh S, Brackney M, et al. Passive multistate surveillance for neutropenia after use of cocaine or heroin possibly contaminated with levamisole. Ann Emerg Med 2013;61:468-74.

14. Duggal HS, Gates C, Pathak PC. Olanzapine-induced neutropenia: mechanism and treatment. J Clin Psychopharmacol 2004;24:234-5.

15. Lim MH, Park JI, Park TW. A case with neutropenia related with the use of various atypical antipsychotics. Psychiatry Investig 2013;10:428-31.

16 Uetrecht J, Zahid N, Tehim A. Structural features associated with reactive metabolite formation in clozapine analogues. Chem Biolo Interact 1997;104:117-29. 